\title{
Patient's age and D-dimer levels predict the prognosis in patients with TAFRO syndrome
}

\author{
Hiroshi Kawabata ${ }^{1}$ (D) Shino Fujimoto ${ }^{1} \cdot$ Tomoyuki Sakai $^{1} \cdot$ Hiroto Yanagisawa ${ }^{1} \cdot$ Toshio Kitawaki $^{2}$. \\ $\mathrm{Kenji} \mathrm{Nara}^{3} \cdot$ Masao Hagihara $^{4} \cdot$ Hiroshi Yamamoto $^{5} \cdot$ Masakuni Tanimizu $^{6}$. Chikako Kato ${ }^{7} \cdot$ Tomoki Origuchi $^{8}$. \\ Kazutaka Sunami ${ }^{9} \cdot$ Yoshitaka Sunami $^{10} \cdot$ Taro Masunari $^{11} \cdot$ Nobuhiko Nakamura $^{12} \cdot$ Masanori Kobayashi $^{13}$. \\ Keiko Yamagami ${ }^{14} \cdot$ Katsuhiro Miura $^{15} \cdot$ Kazue Takai $^{16} \cdot$ Sadao Aoki $^{17} \cdot$ Norifumi Tsukamoto $^{18} \cdot$ Yasufumi Masaki $^{1}$
}

Received: 22 February 2021 / Revised: 22 April 2021 / Accepted: 22 April 2021 / Published online: 30 April 2021

(c) Japanese Society of Hematology 2021, corrected publication 2021

\begin{abstract}
Objectives To identify prognostic factors for TAFRO syndrome, a rare inflammatory disorder of unknown etiology characterized by thrombocytopenia, anasarca, fever, reticulin myelofibrosis, renal dysfunction, and organomegaly.

Methods Data of patients with TAFRO syndrome were extracted from a Japanese patient registry. Patients were divided into groups according to the clinical and laboratory parameters at initial presentation. Cut-off values for the laboratory parameters were determined using receiver operating characteristic curve analysis and by clinical relevance. Patient survival was analyzed by the Kaplan-Meier method. Univariable analysis was performed using log-rank tests. Multivariable analyses were performed with the logistic regression model and the Cox proportional hazards model.

Results We extracted the data of 83 patients with TAFRO syndrome from the registry. Univariable analysis identified several potential prognostic factors. Of these factors, age $\geq 60$ years and D-dimer $\geq 18 \mu \mathrm{g} / \mathrm{dL}$ remained significant predictors of poor overall survival in the multivariable Cox proportional hazards model. Based on these results, we developed a simple prognostic scoring system for TAFRO syndrome (TS-PSS).

Conclusion Patients in our cohort were stratified into low, intermediate, and high-risk groups by the TS-PSS. This system should be verified with independent patient cohorts in future studies.
\end{abstract}

Keywords TAFRO syndrome $\cdot$ Castleman disease $\cdot$ Prognosis $\cdot$ D-dimer

\section{Introduction}

TAFRO syndrome is a rare inflammatory disorder of unknown etiology characterized by thrombocytopenia, anasarca (pleural effusion, ascites, and edema), fever, reticulin myelofibrosis, renal dysfunction, and organomegaly (hepatosplenomegaly and/or lymphadenopathy) $[1,2]$. A large proportion of patients with this syndrome present with generalized lymphadenopathy, and histology of their lymph nodes exhibits features consistent with idiopathic multicentric Castleman disease (iMCD) [3]. Therefore, some researchers assume that this syndrome is a subtype of iMCD (iMCD-TAFRO) [4]. However, the clinical features of this syndrome differ considerably from those of typical

Hiroshi Kawabata

hkawabat@kuhp.kyoto-u.ac.jp

Extended author information available on the last page of the article
iMCD-not otherwise specified (iMCD-NOS). For example, TAFRO syndrome often develops acutely or subacutely, progresses rapidly, and presents with thrombocytopenia without hyper- $\gamma$-globulinemia, while iMCD-NOS usually develops gradually, and presents with thrombocytosis and hyper- $\gamma$-globulinemia $[5,6]$. Therefore, others consider this syndrome a distinct clinical entity [6]; thus, the association between TAFRO syndrome and $\mathrm{MCD}$ is still controversial, especially due to the absence of specific biomarkers for these conditions.

The treatment of TAFRO syndrome encompasses the use of corticosteroids, including methylprednisolone pulse therapy, and other immunosuppressive agents such as tocilizumab, siltuximab, cyclosporine, tacrolimus, rituximab, and rapamycin [7-15]. Despite such immunosuppressive treatments, a third of patients with TAFRO syndrome die within 2 years [6]. International consensus treatment guidelines recommend interleukin 6 (IL-6) blockade (siltuximab 
or tocilizumab) with or without high-dose steroids as the first-line treatment for iMCD-TAFRO [16], but this recommendation was primarily based on a clinical trial that was conducted for entire iMCD patients; the number of enrolled iMCD-TAFRO patients is undescribed [17]. To find an optimal treatment strategy for TAFRO syndrome, we recently analyzed the efficacies of immunosuppressive agents by utilizing a patient registry in Japan [18]. Among 81 patients with TAFRO syndrome, 68 patients received corticosteroids as the first-line treatment, and 47 patients received additional immunosuppressive agents as the second-line treatments. We divided these patients into groups according to the secondline treatments (rituximab, tocilizumab, and cyclosporine A groups) and compared their efficacies based on the primary endpoint set as the time-to-next-treatment or death (TTNT). In this previous study, we found that the TTNT of patients who received rituximab was significantly longer than that of patients who received tocilizumab, although the inter-group differences in overall survival (OS) were not significant [18]. In this cohort, 15 patients who received corticosteroids alone survived beyond 1 year without receiving other immunosuppressive agents. Furthermore, 21 deaths were reported in this cohort, and infections were the most common causes of death. Although serious infections were not common in patients with iMCD treated with siltuximab or tocilizumab $[17,19]$, intensive immunosuppressive therapies theoretically increase risks of serious infections. Therefore, to avoid excessive immunosuppression, risk stratification is crucial to improve the prognosis of patients with this syndrome. In this study, we investigated the prognostic factors for TAFRO syndrome at initial presentation using a retrospective patient database in Japan.

\section{Methods}

\section{Patient cohort}

Since Oct 2013, patients with suspected multicentric Castleman disease (MCD) and TAFRO syndrome have been registered in the Multicenter Collaborative Retrospective Study for Establishing the Concept of TAFRO Syndrome registry (UMIN000011809) from 89 collaborating centers in Japan (Supplementary Table S1). The database includes clinical data at the onset or diagnosis of the condition, histopathological reports, treatments, and outcomes of these patients. We extracted patients with TAFRO syndrome from this database by applying our diagnostic criteria [20]. Briefly, a diagnosis of TAFRO syndrome required all three major categories (anasarca, thrombocytopenia, and systemic inflammation) and at least two of four minor categories (Castleman disease-like features on lymph node biopsy, reticulin myelofibrosis and/or an increased number of megakaryocytes, organomegaly, and progressive renal insufficiency). Patients with human herpesvirus (HHV)-8-associated MCD, POEMS syndrome, common collagen diseases such as systemic lupus erythematosus and Sjögren's syndrome, infectious diseases, and malignancies, including malignant lymphomas were carefully excluded. This process was performed by reviewing the provided data, including autoantibody tests, a minimum of whole body computed tomography scan, and bone marrow biopsy. This study was performed in accordance with the Declaration of Helsinki, and its protocol was approved by the Ethics Committee of Kanazawa Medical University (E183 and I540) as well as the ethics committee of each participating institution.

\section{Statistical analyses}

We identified the following clinical and laboratory parameters as potential prognostic factors: age, sex, presence or absence of iMCD, white blood cell counts (WBC), hemoglobin (Hb), platelet counts (Plt), C-reactive protein (CRP), IL-6, soluble IL-2 receptor (sIL2R), blood urea nitrogen (BUN), creatinine (Cre), lactate dehydrogenase (LDH), aspartate aminotransferase (AST), alanine aminotransferase (ALT), alkaline phosphatase (ALP), $\gamma$-glutamyl transpeptidase ( $\gamma$-GTP), total bilirubin (T-bil), albumin, immunoglobulin $\mathrm{G}$ (IgG), fibrinogen, fibrin/fibrinogen degradation products (FDP), and D-dimer. To determine the cut-off values of these laboratory parameters, we performed receiver operating characteristic (ROC) analysis by setting the dependent variable (outcome) as 1-year survival after initial presentation. Preliminary cut-off points of these laboratory parameters were determined based on the maximum of the Youden index in the ROC curves [21]. We divided our cohort into groups based on these preliminary cut-off values and the median values (round figures were used where appropriate). Final cut-off values were determined based on clinical meaningfulness. The Kaplan-Meier method was used for survival analysis, and log-rank tests were used for comparison between groups (univariable analysis). Multivariable analysis of the potential prognostic factors for TAFRO syndrome was performed using the logistic regression model by setting the outcome as 1-year survival. To confirm independent risk factors for OS, multivariable Cox-proportional hazards model was also performed. Cases with missing data were excluded from the analysis for the related variable. Results with $P$ values less than 0.05 were considered statistically significant. All analyses were performed using EZR, version 1.54 [22].

\section{Results}

\section{Patient characteristics}

In total, 243 patients were registered in the database from Oct 2013 to Jun 2020. Among them, 95 patients were 
diagnosed with TAFRO syndrome, and 83 patients had follow-up data. We restricted this study to these 83 patients. Forty-seven patients were male, and the median age was 50 years (range, 20-85 years). According to the disease severity classification system for TAFRO syndrome wherein scores were calculated by assessing the severities of anasarca, thrombocytopenia, systemic inflammation, and renal damage (Supplementary Table S2) [20], 8, 24, 34, and 12 patients were classified as grades $2,3,4$, and 5, respectively, at the initial presentation, with no patient classified as grade 1 (Table 1). Lymph node biopsy was performed in 66 patients and histology consistent with iMCD was confirmed in 63 patients; these patients fulfilled the diagnostic criteria for HHV-8-negative iMCD (histology of the other 3 patients was nonspecific) [3] (Table 1). In the other 17 patients, lymph node biopsy was not performed due to either the absence of lymphadenopathy or severe hemorrhagic tendency. The median follow-up period for survivors was 53 months. During the follow-up periods, all patients received corticosteroids, including methylprednisolone pulse therapies $(n=43)$. Concerning immunosuppressive treatment, 33 patients received tocilizumab, an anti-IL-6 receptor antibody; 32 patients received cyclosporine; 19 patients received rituximab; 9 patients received cyclophosphamide or combination chemotherapies containing cyclophosphamide; and 1 patient received tacrolimus (some patients sequentially received multiple immunosuppressive therapies). In contrast, non-immunosuppressive treatments encompassed hemodialysis performed in 26 patients, plasma exchange in 11 patients, thrombopoietin receptor agonists in 10 patients, and intravenous immunoglobulin therapy in 2 patients. The 1- and 2-year OS ratios of the entire cohort were $72.0 \%$ and $67.8 \%$, respectively (Fig. 1, panel a).

\section{Univariable analysis}

Univariable analysis was performed for OS with each clinical parameter. There were no significant differences in OS between men and women and between those with iMCD and those without proven iMCD (Table 1). To determine an optimal cut-off point of age, we first divided the patients into 5 age groups: $<40,40-49,50-59,60-69$, and $\geq 70$ years and compared their OS using Kaplan-Meier analysis. OS curves of these groups were significantly different $(P=2.1 \mathrm{e}-06)$, and those of age groups $<40,40-49$, and 50-59 years were superior to those of age groups 60-69 and $\geq 70$ years (Supplementary Fig. S1). Therefore, we set the cut-off age as 60 years. The 1 -year OS of the $<60$ years-old group $(n=57)$ was $89 \%$, while that of the $\geq 60$ years-old group $(n=26)$ was $33 \%$ ( $P=3.1$ e-08, Fig. 1, panel b).

Regarding the laboratory parameters, we explored the optimal cut-off points to use to divide the groups using ROC analysis, as described in the method section (Supplementary
Table S3 and Supplementary Fig. S2). Round figures around these values or median values were set as preliminary cutoff points. We determined the final cut-off points considering the clinical meaningfulness (Supplementary Table S4). For example, for AST, ALT, LDH, T-bil, and IgG, both the preliminary cut-off value calculated by the ROC analysis and the median value were within reference ranges; hence, round figures just on or above the upper limits of these reference ranges were used as the final cut-off points. Patients were divided into two groups according to these points, and survival was compared using the Kaplan-Meier method. Among laboratory parameters, WBC, CRP, sIL2R, ALT, LDH, ALP, and $\gamma$-GTP did not significantly correlate with OS (Table 1). In contrast, $\mathrm{Hb}<8 \mathrm{~g} / \mathrm{dL}$, platelet counts $<30 \times 10^{3} / \mu \mathrm{L}, \mathrm{BUN} \geq 40 \mathrm{mg} / \mathrm{dL}$, creatinine $\geq 2 \mathrm{mg} /$ $\mathrm{dL}, \mathrm{AST} \geq 35 \mathrm{U} / \mathrm{L}$, albumin $<2.5 \mathrm{~g} / \mathrm{dL}, \mathrm{IgG} \geq 1700 \mathrm{mg} / \mathrm{dL}$, fibrinogen $<450 \mathrm{mg} / \mathrm{dL}, \mathrm{FDP} \geq 10 \mu \mathrm{g} / \mathrm{dL}, \mathrm{D}$-dimer $\geq 18 \mu \mathrm{g} /$ $\mathrm{dL}$, and IL- $6 \geq 21 \mathrm{pg} / \mathrm{mL}$ were significantly associated with inferior OS in univariable analysis (Table 1; the results of the representative variables are also shown in Fig. 1, panels $\mathrm{c}-\mathrm{f}$ ). We tested whether the disease severity classification system could predict patient's prognosis using Kaplan-Meier analysis with the log-rank tests. The OS curves for patients with grades 2, 3, and 4 were not distinct, but the OS curve of the patients with grade 5 was significantly inferior to that of the other grades when combined (Fig. 1, panels $g$ and $h$ ).

\section{Multivariable analyses}

Next, we explored independent prognostic factors using the multivariable logistic regression model by setting the outcome as 1-year survival. Factors that showed significant association with survival in univariable analysis were candidates to incorporate into this analysis. However, considering the low number of events $(n=26)$, to avoid overfitting, only a couple of variables could be incorporated. We considered that five variables with the most significant inter-group differences in the univariable analysis shown in Table $1(P<0.01$, i.e., age, Hb, AST, fibrinogen, and D-dimer) were candidates. Among them, we decided to select three variables by considering their clinical relevance. D-dimer is a degenerative product of fibrinogen, and both are related to coagulation status. Because D-dimer showed a smaller $P$ value than fibrinogen, we chose D-dimer as a representative candidate of coagulation markers and discarded fibrinogen in the multivariable analysis. All of four parameters that could be associated with coagulopathy (PLT, fibrinogen, FDP, and D-dimer) showed significant association with OS in the univariable analysis (Table 1), indicative of the importance of coagulation status on patient prognosis. Next, we questioned whether AST is a reasonable variable to incorporate into the multivariable analysis, because both its preliminary 
Table 1 Univariable analysis for overall survival

\begin{tabular}{|c|c|c|c|c|c|}
\hline $\begin{array}{l}\text { Categories (unit) [refer- } \\
\text { ence ranges] }\end{array}$ & Median $(25-75 \% o)$ & Criteria & $n$ & $\begin{array}{l}\text { One year survival } \\
\%(95 \% \mathrm{CI})\end{array}$ & $P$ value \\
\hline \multirow[t]{3}{*}{ Age (years) } & $50(44-62.5)$ & & 83 & & \\
\hline & & $<60$ years & 57 & 89.4 [77.9-95.1] & \multirow[t]{2}{*}{$3.1 \times 10^{-8}$} \\
\hline & & $\geq 60$ years & 26 & $33.1[16.0-51.4]$ & \\
\hline \multirow[t]{2}{*}{ Gender } & & Male & 47 & $65.6[50.0-77.3]$ & \multirow[t]{2}{*}{0.34} \\
\hline & & Female & 36 & 80.6 [63.5-90.2] & \\
\hline \multirow[t]{2}{*}{ Castleman disease } & & With iMCD & 63 & 71.0 [58.0-80.7] & \multirow[t]{2}{*}{0.90} \\
\hline & & Without iMCD & 20 & $75.0[50.0-88.7]$ & \\
\hline \multirow{3}{*}{$\begin{array}{l}\mathrm{WBC}\left(\times 10^{3} / \mu \mathrm{L}\right) \\
{[3.3-8.6]}\end{array}$} & $9.4(7.0-13.0)$ & & 83 & & \\
\hline & & $<12.0$ & 55 & 77.8 [64.2-86.7] & \multirow[t]{2}{*}{0.08} \\
\hline & & $\geq 12.0$ & 28 & $60.7[40.4-76.0]$ & \\
\hline \multirow{3}{*}{$\begin{array}{l}\text { Hemoglobin }(\mathrm{g} / \mathrm{dL}) \\
\text { [Male: } 13.7-16.8 \\
\text { Female: } 11.6-14.8]\end{array}$} & $9.6(7.4-11.5)$ & & 83 & & \multirow{3}{*}{0.0073} \\
\hline & & $<8$ & 26 & 57.7 [36.8-73.9] & \\
\hline & & $\geq 8$ & 57 & $78.6[65.5-87.3]$ & \\
\hline \multirow{3}{*}{$\begin{array}{l}\text { Platelet counts } \\
\left(\times 10^{3} / \mu \mathrm{L}\right) \\
{[158-343]}\end{array}$} & $33(17-56)$ & & 83 & & \multirow{3}{*}{0.034} \\
\hline & & $<30$ & 37 & 64.6 [46.9-77.7] & \\
\hline & & $\geq 30$ & 46 & $78.0[63.0-87.5]$ & \\
\hline \multirow{3}{*}{$\begin{array}{l}\text { CRP }(\mathrm{mg} / \mathrm{dL}) \\
{[\leq 0.1]}\end{array}$} & $16.2(6.8-22.6)$ & & 83 & & \multirow{3}{*}{0.49} \\
\hline & & $<25$ & 66 & $70.8[58.1-80.3]$ & \\
\hline & & $\geq 25$ & 17 & 76.5 [48.8-90.4] & \\
\hline \multirow[t]{3}{*}{ BUN (mg/dL) [8-20] } & $32(21-60)$ & & 82 & & \\
\hline & & $<40$ & 47 & $82.8[68.5-91.0]$ & \multirow[t]{2}{*}{0.015} \\
\hline & & $\geq 40$ & 35 & $56.8[38.8-71.3]$ & \\
\hline \multirow{3}{*}{$\begin{array}{l}\text { Creatinine } \\
\text { (mg/dL) } \\
\text { [Male: } 0.65-1.07 \\
\text { Female: } 0.46-0.79]\end{array}$} & $1.50(1.06-2.10)$ & & 83 & & \multirow{3}{*}{0.039} \\
\hline & & $<2$ & 58 & $79.0[66.0-87.5]$ & \\
\hline & & $\geq 2$ & 25 & $56.0[34.8-72.7]$ & \\
\hline \multirow[t]{3}{*}{ AST (U/L) [13-30] } & $23.5(18-35)$ & & 82 & & \\
\hline & & $<35$ & 61 & 81.7 [69.4-89.4] & \multirow[t]{2}{*}{0.00035} \\
\hline & & $\geq 35$ & 21 & $42.9[21.9-62.3]$ & \\
\hline \multirow{3}{*}{$\begin{array}{l}\text { ALT (U/L) } \\
\text { [Male: 10-42 } \\
\text { Female: 7-23] }\end{array}$} & $17.5(11-29)$ & & 82 & & \\
\hline & & $<45$ & 71 & $75.8[64.0-84.2]$ & 0.064 \\
\hline & & $\geq 45$ & 11 & $63.1[16.7-70.7]$ & \\
\hline LDH (U/L) & 217 (181-283) & & 82 & & \\
\hline$[124-222]$ & & $<230$ & 47 & 74.5 [59.4-84.6] & 0.62 \\
\hline & & $\geq 230$ & 35 & $67.9[49.5-80.8]$ & \\
\hline Alkaline phosphatase & $537(375-1116)$ & & 82 & & \\
\hline (U/L) & & $<420$ & 31 & 83.9 [65.5-92.9] & 0.13 \\
\hline$[100-522]$ & & $\geq 420$ & 51 & 64.1 [49.1-75.7] & \\
\hline$\gamma$-GTP (U/L) & $81(46-160)$ & & 79 & & \\
\hline [Male: 13-64 & & $<70$ & 34 & $67.3[47.8-80.4]$ & 0.31 \\
\hline Female: 9-32] & & $\geq 70$ & 45 & $73.1[57.5-83.7]$ & \\
\hline Total bilirubin & $0.8(0.6-1.1)$ & & 81 & & \\
\hline$(\mathrm{mg} / \mathrm{dL})$ & & $<1.5$ & 63 & $76.0[63.4-84.8]$ & 0.15 \\
\hline$[0.4-1.5]$ & & $\geq 1.5$ & 18 & $54.5[29.2-74.2]$ & \\
\hline Albumin $(\mathrm{g} / \mathrm{dL})$ & $2.3(1.9-2.65)$ & & 83 & & \\
\hline$[4.1-5.1]$ & & $<2.5$ & 56 & 63.8 [49.6-74.9] & 0.024 \\
\hline & & $\geq 2.5$ & 27 & 88.9 [69.4-96.3] & \\
\hline $\operatorname{IgG}(\mathrm{mg} / \mathrm{dL})$ & 1318 (1092-1694) & & 79 & & \\
\hline [861-1747] & & $<1700$ & 59 & $79.5[66.7-87.8]$ & 0.010 \\
\hline & & $\geq 1700$ & 20 & $50.0[27.1-69.2]$ & \\
\hline
\end{tabular}


Table 1 (continued)

\begin{tabular}{|c|c|c|c|c|c|}
\hline $\begin{array}{l}\text { Categories (unit) [refer- } \\
\text { ence ranges] }\end{array}$ & Median (25-75\%o) & Criteria & $n$ & $\begin{array}{l}\text { One year survival } \\
\%(95 \% \mathrm{CI})\end{array}$ & $P$ value \\
\hline \multirow{3}{*}{$\begin{array}{l}\text { Fibrinogen }(\mathrm{mg} / \mathrm{dL}) \\
{[170-410]}\end{array}$} & $483(406-637)$ & & 77 & & \\
\hline & & $<450$ & 28 & 59.5 [38.8-75.3] & 0.0086 \\
\hline & & $\geq 450$ & 49 & 79.6 [65.4-88.5] & \\
\hline \multirow{3}{*}{$\begin{array}{l}\text { FDP }(\mu \mathrm{g} / \mathrm{mL}) \\
{[\leq 5.0]}\end{array}$} & $23.4(14.2-42.5)$ & & 62 & & \\
\hline & & $<10$ & 8 & NA [NA-NA] & 0.045 \\
\hline & & $\geq 10$ & 54 & $64.2[49.8-75.5]$ & \\
\hline \multirow{3}{*}{$\begin{array}{l}\text { D-dimer }(\mu \mathrm{g} / \mathrm{mL}) \\
{[\leq 1.0]}\end{array}$} & $11.8(5.9-19.8)$ & & 64 & & \\
\hline & & $<18$ & 45 & 84.3 [69.8-92.2] & 0.0018 \\
\hline & & $\geq 18$ & 19 & $51.3[27.1-71.1]$ & \\
\hline \multirow{3}{*}{$\begin{array}{l}\mathrm{IL}-6(\mathrm{pg} / \mathrm{mL}) \\
{[\leq 4.0]}\end{array}$} & $25.4(13.7-35.8)$ & & 66 & & \\
\hline & & $<21$ & 25 & 88.0 [67.3-96.0] & 0.047 \\
\hline & & $\geq 21$ & 41 & $62.9[46.1-75.7]$ & \\
\hline \multirow{3}{*}{$\begin{array}{l}\text { sIL2R (U/mL) } \\
{[157-474]}\end{array}$} & $1702(1133-2435)$ & & 78 & & \\
\hline & & $<2500$ & 62 & 73.9 [60.9-83.1] & 0.31 \\
\hline & & $\geq 2500$ & 16 & $56.2[29.5-72.6]$ & \\
\hline \multirow[t]{4}{*}{ Disease severity } & & Grade 2 & 8 & 87.5 [38.7-98.1] & 0.022 \\
\hline & & Grade 3 & 27 & 74.1 [53.2-86.7] & \\
\hline & & Grade 4 & 34 & 79.0 [60.8-89.4] & \\
\hline & & Grade 5 & 12 & $41.7[15.2-66.5]$ & \\
\hline
\end{tabular}

Representative reference ranges are shown because the ranges varied slightly depending on institution. $P$ values less than 0.05 are shown in bold

Abbreviations: $W B C$ white blood cell count, $C R P$ C-reactive protein, $I L-6$ interleukin $6, B U N$ blood urea nitrogen, $L D H$ lactate dehydrogenase, $A S T$ aspartate aminotransferase, $A L T$ alanine aminotransferase, $\gamma$-GTP $\gamma$-glutamyl transpeptidase, $I g G$ immunoglobulin $\mathrm{G}, i M C D$ idiopathic multicentric Castleman disease, $N A$ not applicable

cut-off value calculated by ROC analysis (27 U/L) and median value $(23.5 \mathrm{U} / \mathrm{L})$ were within its reference range, AST $>35 \mathrm{U} / \mathrm{L}$ can be nonspecifically observed in apparently healthy individuals, its area under the curve in ROC analysis was relatively small (0.6254, Supplementary Table S3 and Supplementary Fig. S2), and, moreover, none of the other markers of liver (and/or tissue) damage, i.e., LDH, ALT, $\gamma$-GTP, ALP, and T-bil, were significantly associated with survival in univariable analysis (Table 1). Therefore, we decided not to incorporate AST into the multivariable analysis, and chose the remaining 3 variables, i.e., age, D-dimer, and $\mathrm{Hb}$. Our variable selection was supported by the fact that, when we preliminarily included all these 5 candidate variables into the multivariable logistic regression model, stepwise variable selection based on Bayesian information criterion also eliminated AST and fibrinogen. Accordingly, age, Hb, and D-dimer were identified as significant prognostic factors associated with 1-year survival in the final model (Table 2). When we included these 3 factors into the Cox-proportional hazards model for OS, only age $\geq 60$ years and D-dimer $\geq 18 \mu \mathrm{g} /$ $\mathrm{mL}$ remained significant prognostic factors (Table 3 ).

\section{Development of a prognostic scoring system for TAFRO syndrome}

Based on the multivariable analysis shown in Table 3, a simple clinical scoring system was developed by assigning one point each to age $\geq 60$ years and D-dimer $\geq 18 \mu \mathrm{g} / \mathrm{dL}$. The values of D-dimer at initial presentation were available in only 64 patients; we classified these patients into 3 groups based on the total scores of 0,1 , and 2 for low, intermediate and high-risk groups, respectively, and analyzed their OS using the Kaplan-Meier method. The OS curves for the low $(n=35)$, intermediate $(n=22)$, and high-risk $(n=7)$ groups were clearly distinct, with the 1-year survival ratios of 97.1, 54.5 , and $21.4 \%$, respectively $\left(P=1.0 \times 10^{-7}\right.$; Fig. 2$)$.

\section{Discussion}

In this study, we investigated potential prognostic factors for patients with TAFRO syndrome using a Japanese patient database and found a number of factors that were associated with patient survival in univariable analysis. Among them, age $\geq 60$ years, $\mathrm{Hb}<8 \mathrm{~g} / \mathrm{dL}, \mathrm{AST} \geq 35 \mathrm{U} / \mathrm{L}$, 
a

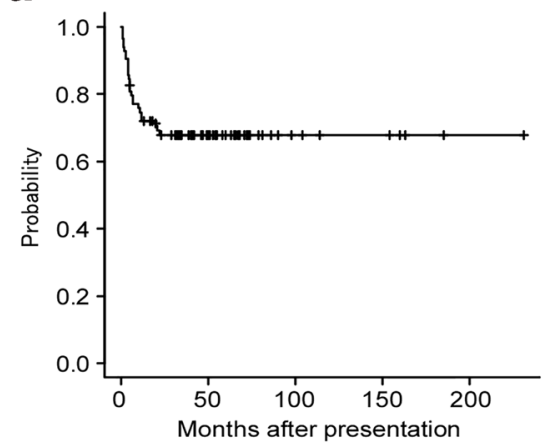

d

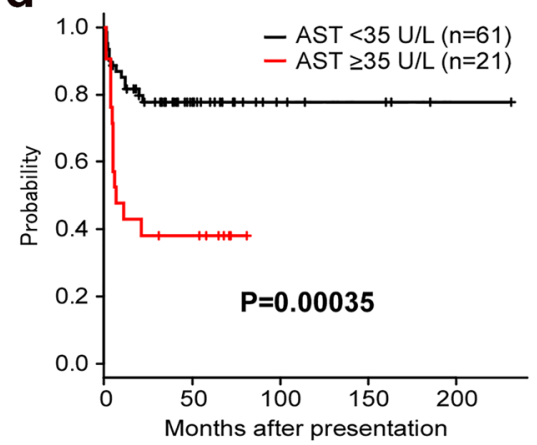

g

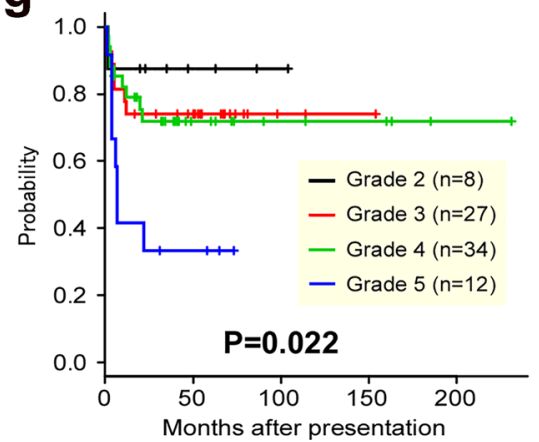

b

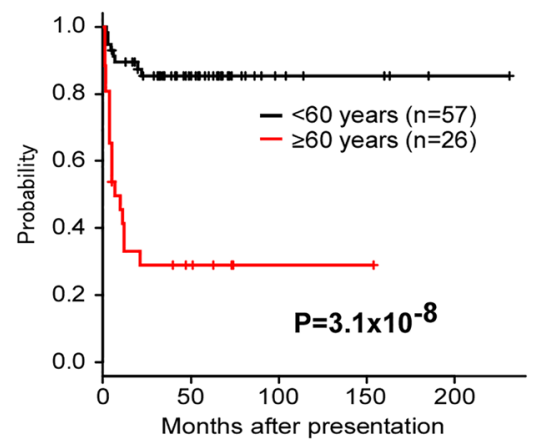

e

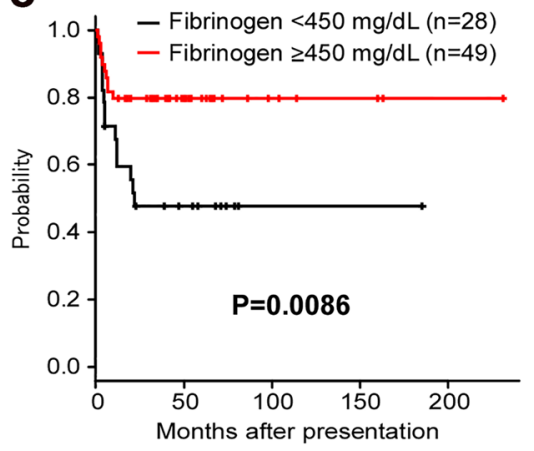

h

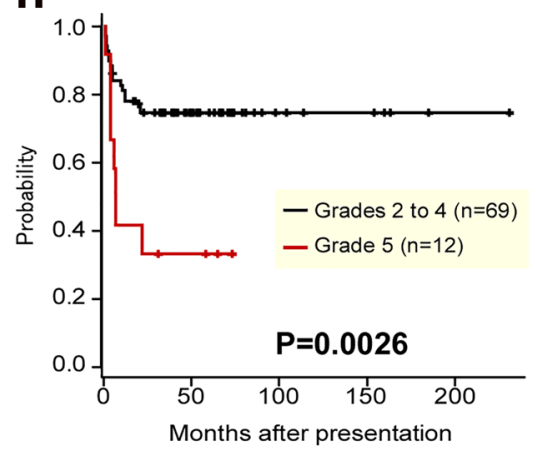

C

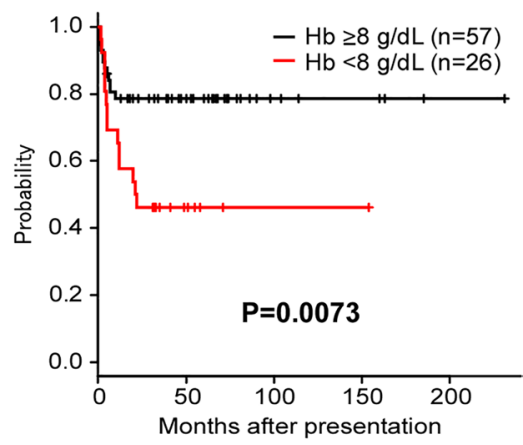

f

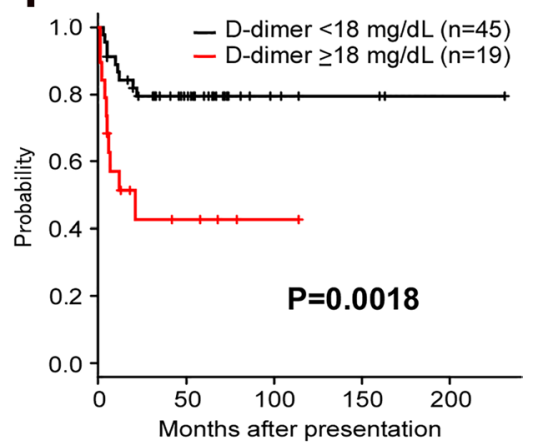

Fig. 1 Overall survival after the initial presentation in patients with TAFRO syndrome according to their clinical parameters. a The survival curve of the entire cohort. b-g Comparison of survival curves between groups according to their $\mathbf{b}$ age, $\mathbf{c}$ hemoglobin $(\mathrm{Hb})$, d

Table 2 Multivariable analysis for 1-year survival

\begin{tabular}{lcll}
\hline Categories & Odds ratio & $95 \% \mathrm{CI}$ & $P$ value \\
\hline Age $\geq 60$ years & 22.7 & $4.0-129.0$ & 0.00041 \\
D-dimer $\geq 18 \mu \mathrm{g} / \mathrm{mL}$ & 9.9 & $1.8-55.4$ & 0.0090 \\
$\mathrm{Hb}<8 \mathrm{~g} / \mathrm{dL}$ & 6.3 & $1.3-31.8$ & 0.025 \\
\hline
\end{tabular}

Logistic regression model was employed

$\mathrm{Hb}$ hemoglobin, CI confidence interval aspartate aminotransferase (AST), e fibrinogen, $\mathbf{f}$ D-dimer, and $\mathbf{g}, \mathbf{h}$ disease severity determined by the method shown in Supplementary Table S2. Survival of the patients was compared using the KaplanMeier method with log-rank tests

fibrinogen $<450 \mathrm{mg} / \mathrm{dL}$, and D-dimer $\geq 18 \mu \mathrm{g} / \mathrm{dL}$ were significantly associated with poor OS at $P$ values less than 0.01 . Among these variables, ages, $\mathrm{Hb}$, and D-dimer were chosen as clinically relevant variables to incorporate into multivariable analyses. All of these 3 were significantly associated with 1-year survival in the multivariable logistic regression model. Furthermore, age $\geq 60$ years and D-dimer $\geq 18 \mu \mathrm{g} /$ $\mathrm{dL}$ remained as statistically significant predictors of poor OS in the Cox-proportional hazards model. Applying the latter results, we developed a simple prognostic scoring system for TAFRO syndrome (TS-PSS). Using this system, patients in our cohort were stratified into low, intermediate, 
Table 3 Multivariable analysis for overall survival

\begin{tabular}{lllll}
\hline Categories & Hazard ratio & $95 \% \mathrm{CI}$ & $P$ value & $\begin{array}{l}\text { Prog- } \\
\text { nostic } \\
\text { score }\end{array}$ \\
\hline Age $\geq 60$ years & 5.49 & $1.98-15.19$ & 0.0010 & 1 \\
$\mathrm{D}-\mathrm{dimer} \geq 18 \mu \mathrm{g} / \mathrm{mL}$ & 3.19 & $1.272-8.04$ & 0.014 & 1 \\
$\mathrm{Hb}<8 \mathrm{~g} / \mathrm{dL}$ & 1.80 & $0.68-4.77$ & 0.24 & \\
\hline
\end{tabular}

Cox-proportional hazards model was employed. Based on the results shown in this table, the prognostic score of 1 each was assigned for age $\geq 60$ years and D-dimer $\geq 18 \mu \mathrm{g} / \mathrm{mL}$, and patients with total scores of 0,1 , and 2 were classified into low-, intermediate-, and high-risk groups, respectively

$H b$ hemoglobin, $C I$ confidence interval

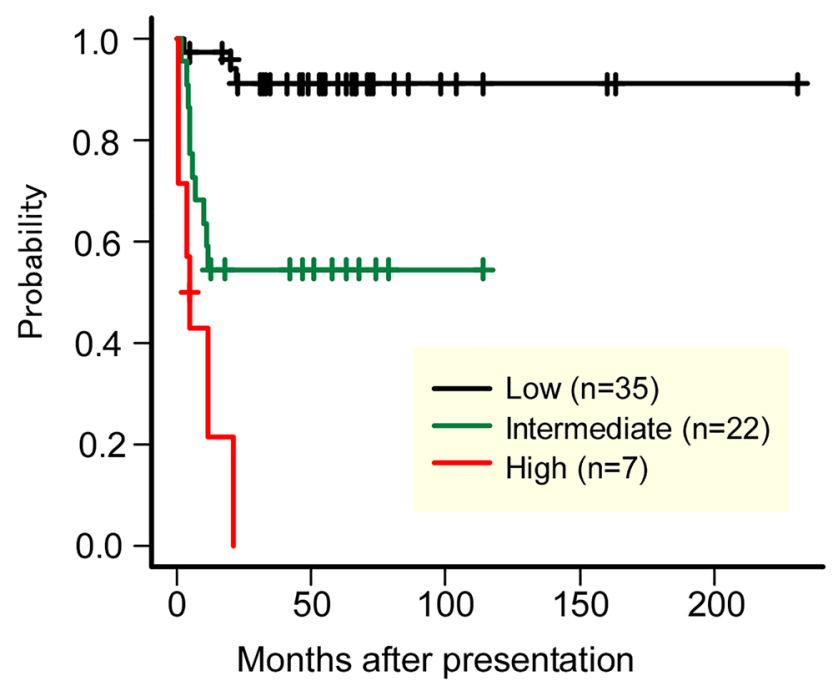

Fig. 2 Overall survival after the initial presentation in patients with TAFRO syndrome according to risk groups stratified by the TS-PSS system. Survival of the patients was compared using the KaplanMeier method with log-rank tests

and high-risk groups. Most patients were classified into the low-risk group with a favorable prognosis. In contrast, the prognosis of those in the high-risk group was dismal, hence the need to develop novel therapeutic strategies for them. The prognosis of patients in the intermediate-risk group was variable; it may be changeable depending on therapeutic interventions.

Of the prognostic factors identified in this study, age $\geq 60$ years was the strongest predictor of poor OS; this observation was reasonable because it probably reflects the reduction in reserve capacities of various organs and vascular aging. Notably, only one-third of patients aged $\geq 60$ years survived beyond 12 months in our cohort.

Elevation of plasma D-dimer levels was another predictor for the survival of patients with TAFRO syndrome in multivariable analysis. We previously reported significantly higher plasma FDP and D-dimer levels in patients with TAFRO syndrome than in those with iMCD-NOS [6]. $\mathrm{D}$-dimer is a component of FDP, and its elevation in plasma is usually associated with coagulopathies such as disseminated intravascular coagulation (DIC) and other thrombotic or microthrombotic events. Because simultaneous measurements of D-dimer and FDP were not approved under the Japanese health insurance system, only one of these values was available in some patients. Despite the missing data, D-dimer was identified as statistically significant prognostic factors for poor OS, indicating the significant involvement of coagulopathy in TAFRO syndrome. Significantly poor OS in patients with relatively low fibrinogen levels $(<450 \mu \mathrm{g} / \mathrm{dL})$ notwithstanding systemic inflammation, might also reflect the activation of the coagulation system in which fibrinogen was consumed. Critically ill patients with TAFRO syndrome may have coagulopathy with microangiopathy, similar to those with severe COVID-19 infection [23]. Indeed, thrombotic microangiopathy was demonstrated in renal biopsy specimens in patients with TAFRO syndrome [24-28].

Anemia $(\mathrm{Hb}<8 \mathrm{~g} / \mathrm{dL})$ was also selected as a significant risk factor for 1-year mortality in the logistic regression model, though it failed to show statistical significance in Cox-proportional hazards model for OS. Anemia observed in TAFRO syndrome may be multifactorial, but it is probably associated with inflammation. The major mechanism of anemia of inflammation is based on the restriction of available iron through overproduction of hepcidin, the master regulator of iron homeostasis, induced by IL-6 [29]. Relatively decreased production of erythropoietin caused by renal damage, and shortening of erythrocyte life-span, may also be involved [29].

Elevation of $\mathrm{AST} \geq 35 \mathrm{U} / \mathrm{L}$ was also found to be a predictor of poor OS with a small $\mathrm{P}$ value in the univariable analysis. This result is rather incomprehensible because the cut-off value for AST ( $35 \mathrm{U} / \mathrm{L}$ ) was just above the reference range, and none of the other parameters for hepatic damage were significantly associated with OS in the univariable analysis. Based on clinical considerations, we assumed that the small $P$ value observed for AST in the univariable analysis might be incidental, and we eliminated AST from the multivariable analyses. Notably, the elevation of AST is less specific than that of ALT for liver diseases; as it can be observed in cardiac, skeletal muscle, kidney, erythrocytes, and other tissue damages [30]. Given the nature of the registry, the elevated AST levels in patients with poor OS might have reflected unreported comorbidities. Thus, the clinical significance of AST $\geq 35 \mathrm{U} / \mathrm{L}$ in this syndrome is still uncertain.

We previously proposed the disease severity classification system wherein disease severity was classified based on the severity of anasarca, fever/CRP, thrombocytopenia, and renal damage (Supplementary Table S2) [20]. Anasarca 
is closely related to hypoalbuminemia, and renal damage is associated with serum BUN and Cre levels. Therefore, from its definition, the disease severity score should be correlated with CRP and BUN/Cre, and inversely correlated with albumin and PLT. We did not incorporate disease severity score into the multivariable analyses because it is closely related to these laboratory parameters from its definition. However, among these parameters, albumin, PLT, BUN, and Cre were significantly associated with OS in univariable analysis with $P$ values between 0.05 and 0.01 (Table 1). In fact, the OS of patients with disease severity grade 5 was significantly inferior to that of those with grades 4 or less, when combined, as shown in new Fig. 1, panel h. Therefore, the disease severity score at presentation may still be useful to identify those with very poor prognosis.

Our study has several limitations. First, the patients were not prospectively registered but registered in many institutes, which might have caused selection bias and possible inaccuracy in data. Second, because TAFRO syndrome usually progresses rapidly and the patient's condition deteriorates quickly, their laboratory data change dynamically. Though we used patient's data at their initial presentation, the timings of data collection in their clinical courses might have been diverse, and these might have affected the results of this study. Third, the numbers of enrolled patients and events were small; therefore, we could include only a few parameters in the multivariable analyses. In addition, some of the clinical parameters such as FDP and D-dimer values were missing at presentation in a considerable number of cases, which further reduced the statistical power. Fourth, although the diagnosis of TAFRO syndrome was centrally reviewed, other diseases, such as infectious, autoimmune, and malignant diseases, could be misdiagnosed for this syndrome due to the lack of specific diagnostic markers. Fifth, treatment strategies were not standardized; instead, they were chosen by attending physicians in each institute. However, because of the rarity of TAFRO syndrome [31], conducting a largescale prospective study on this syndrome is unrealistic at this moment, unfortunately.

In conclusion, although there were several limitations, we revealed that older age and raised D-dimer levels at initial presentation were predictors of poor OS based on limited resources. Utilizing these results, we have, for the first time, developed a prognostic scoring system, TS-PSS, which could stratify patients with TAFRO syndrome into 3 risk groups. This system may allow physicians to assess prognosis earlier, when they first suspect this intractable disorder. Prognostic prediction is quite important in identifying those who require intensive treatments and closer monitoring. With independent patient cohorts, this system should be verified in future studies. Further, the development of novel therapeutic strategies for high-risk patients requires urgent attention.
Supplementary Information The online version contains supplementary material available at https://doi.org/10.1007/s12185-021-03159-x.

Acknowledgements The authors thank the patients, their families, all the investigators, and nurses in the collaborating centers of this study listed in the Supplementary Table S1. We would like to thank Editage (www.editage.com) for English language editing.

Author's contributions All authors participated in the clinical enrollment/work-up of the patients. H.K. and S.F. designed the research and analyzed the data. H.K. reviewed the literature and wrote the manuscript. Y.M. supervised the research and reviewed the study results. All authors read and approved the final manuscript.

Funding This work was partially supported by the Research Program of Intractable Disease provided by the Ministry of Health, Labor, and Welfare (MHLW) of Japan (H27-28 Nanchi, etc. (Nan)- General-002; H27-Nanchi, etc. (Nan)-General-008; H29-Nanchi, etc. (Nan)General-008; H29-Nanchi, etc. (Nan)- General-019; H29-Nanchi, etc. (Nan)-General-058), and by the Ministry of Education, Culture, Sports, Science and Technology (Grant Nos.17591060 and 15K09510), the Kanazawa Medical University Research Foundation (Grant Nos. S2004-16 and S2007-5), Grant for Assist KAKEN from Kanazawa Medical University (Grant No.K2011-7), Grant for Project Research from High-Tech Research Center of Kanazawa Medical University (Grant No.H2011-11) and Grant for Alumni Research (A) from Kanazawa Medical University (AR2012-06).

\section{Declarations}

Conflict of interest Dr. KAWABATA reports personal fees from Kyowa Hakko Kirin Co., Ltd., Chugai Pharmaceutical Co., Ltd., Novartis Pharma K.K., and Nippon Shinyaku Co., Ltd. outside the submitted work; Dr. K. SUNAMI reports grants from Ono Pharmaceutical Co. Ltd., MSD K. K., Celgene Corporation, AbbVie GK., Takeda Pharmaceutical Co., Ltd., Sanofi K.K., Bristol-Myers Squibb, Daiichi Sankyo Co. Ltd., Alexion Pharmaceuticals Inc., GlaxoSmithKline K. K., Novartis Pharma K.K., and Janssen Pharmaceutical K.K., and personal fees from Celgene Corporation, Ono Pharmaceutical Co. Ltd., BristolMyers Squibb, and Takeda Pharmaceutical Co., Ltd. outside the submitted work; Dr. Tsukamoto reports personal fees from Kyowa Hakko Kirin Co., Ltd. outside the submitted work; Dr. AOKI reports personal fees from AbbVie GK. and Janssen Pharmaceutical K.K. outside the submitted work; Dr. Masaki reports grants from Eisai Co., Ltd., Kyowa Hakko Kirin Co., Ltd., Astellas Pharma Inc., Ono Pharmaceutical Co. Ltd., and Pfizer Seiyaku K. K. outside the submitted work.

\section{References}

1. Takai K, Nikkuni K, Shibuya H, Hashidate H. Thrombocytopenia with mild bone marrow fibrosis accompanied by fever, pleural effusion, ascites and hepatosplenomegaly. Rinsho Ketsueki. 2010;51:320-5.

2. Kawabata H, Takai K, Kojima M, Nakamura N, Aoki S, Nakamura $\mathrm{S}$, et al. Castleman-Kojima disease (TAFRO syndrome): A novel systemic inflammatory disease characterized by a constellation of symptoms, namely, thrombocytopenia, ascites (anasarca), microcytic anemia, myelofibrosis, renal dysfunction, and organomegaly: a status report and summary of Fukushima (6 June, 2012) and Nagoya meetings (22 September, 2012). J Clin Exp Hematop. 2013;53:57-61. 
3. Fajgenbaum DC, Uldrick TS, Bagg A, Frank D, Wu D, Srkalovic $\mathrm{G}$, et al. International, evidence-based consensus diagnostic criteria for HHV-8-negative/idiopathic multicentric Castleman disease. Blood. 2017;129:1646-57.

4. Dispenzieri A, Fajgenbaum DC. Overview of Castleman disease. Blood. 2020;135:1353-64.

5. Iwaki N, Fajgenbaum DC, Nabel CS, Gion Y, Kondo E, Kawano $\mathrm{M}$, et al. Clinicopathologic analysis of TAFRO syndrome demonstrates a distinct subtype of HHV-8-negative multicentric Castleman disease. Am J Hematol. 2016;91:220-6.

6. Fujimoto S, Sakai T, Kawabata H, Kurose N, Yamada S, Takai $\mathrm{K}$, et al. Is TAFRO syndrome a subtype of idiopathic multicentric Castleman disease? Am J Hematol. 2019;94:975-83.

7. Iwaki N, Sato Y, Takata K, Kondo E, Ohno K, Takeuchi M, et al. Atypical hyaline vascular-type Castleman's disease with thrombocytopenia, anasarca, fever, and systemic lymphadenopathy. J Clin Exp Hematop. 2013;53:87-93.

8. Kawabata H, Kotani S, Matsumura Y, Kondo T, Katsurada T, Haga H, et al. Successful treatment of a patient with multicentric Castleman's disease who presented with thrombocytopenia, ascites, renal failure and myelofibrosis using tocilizumab, an antiinterleukin-6 receptor antibody. Intern Med. 2013;52:1503-7.

9. Masaki Y, Nakajima A, Iwao H, Kurose N, Sato T, Nakamura T, et al. Japanese variant of multicentric castleman's disease associated with serositis and thrombocytopenia-a report of two cases: is TAFRO syndrome (Castleman- Kojima disease) a distinct clinicopathological entity? J Clin Exp Hematop. 2013;53:79-85.

10. Kubokawa I, Yachie A, Hayakawa A, Hirase S, Yamamoto N, Mori T, et al. The first report of adolescent TAFRO syndrome, a unique clinicopathologic variant of multicentric Castleman's disease. BMC Pediatr. 2014;14:139.

11. Konishi Y, Takahashi S, Nishi K, Sakamaki T, Mitani S, Kaneko $\mathrm{H}$, et al. Successful treatment of TAFRO syndrome, a variant of multicentric Castleman's disease, with cyclosporine A: possible pathogenetic contribution of interleukin-2. Tohoku J Exp Med. 2015;236:289-95.

12. Tedesco S, Postacchini L, Manfredi L, Goteri G, Luchetti MM, Festa A, et al. Successful treatment of a Caucasian case of multifocal Castleman's disease with TAFRO syndrome with a pathophysiology targeted therapy-a case report. Exp Hematol Oncol. 2015;4:3.

13. Fujiwara S, Mochinaga $\mathrm{H}$, Nakata H, Ohshima K, Matsumoto M, Uchiba M, et al. Successful treatment of TAFRO syndrome, a variant type of multicentric Castleman disease with thrombotic microangiopathy, with anti-IL-6 receptor antibody and steroids. Int J Hematol. 2016;103:718-23.

14. Fajgenbaum DC, Langan RA, Japp AS, Partridge HL, Pierson SK, Singh A, et al. Identifying and targeting pathogenic PI3K/AKT/ mTOR signaling in IL-6-blockade-refractory idiopathic multicentric Castleman disease. J Clin Invest. 2019;129:4451-63.

15. Miatech JL, Patel NR, Latuso NQ, Ellipeddi PK. TAFRO syndrome: a case of significant endocrinopathy in a Caucasian patient. Cureus. 2019;11:e4946.

16. van Rhee F, Voorhees P, Dispenzieri A, Fossa A, Srkalovic G, Ide $\mathrm{M}$, et al. International, evidence-based consensus treatment guidelines for idiopathic multicentric Castleman disease. Blood. 2018;132:2115-24.
17. van Rhee F, Wong RS, Munshi N, Rossi JF, Ke XY, Fossa A, et al. Siltuximab for multicentric Castleman's disease: a randomised, double-blind, placebo-controlled trial. Lancet Oncol. 2014;15:966-74.

18. Fujimoto S, Kawabata H, Sakai T, Yanagisawa H, Nishikori M, Nara K, et al. Optimal treatments for TAFRO syndrome: a retrospective surveillance study in Japan. Int $\mathbf{J}$ Hematol. 2021;113:73-80.

19. Murakami M, Johkoh T, Hayashi S, Ohshima S, Mizuki M, Nakatsuka SI, et al. Clinicopathologic characteristics of 342 patients with multicentric Castleman disease in Japan. Mod Rheumatol. 2020;30:843-51.

20. Masaki Y, Kawabata H, Takai K, Tsukamoto N, Fujimoto S, Ishigaki Y, et al. 2019 Updated diagnostic criteria and disease severity classification for TAFRO syndrome. Int J Hematol. 2020;111:155-8.

21. Akobeng AK. Understanding diagnostic tests 3: receiver operating characteristic curves. Acta Paediatr. 2007;96:644-7.

22. Kanda Y. Investigation of the freely available easy-to-use software "EZR" for medical statistics. Bone Marrow Transplant. 2013;48:452-8.

23. Mahajan P, Dass B, Radhakrishnan N, McCullough PA. COVID19-associated systemic thromboembolism: a case report and review of the literature. Cardiorenal Med. 2020;10:462-9.

24. Saito H, Tanaka K, Fujiwara M, Iwasaki T, Numata T, Oda A, et al. Pathological findings of progressive renal involvement in a patient with TAFRO syndrome. CEN Case Rep. 2019;8:239-45.

25. Nagayama Y, Yamano M, Yagame M, Nariyama T, Takahashi M, Kawamoto M, et al. TAFRO syndrome as a cause of glomerular microangiopathy: a case report and literature review. BMC Nephrol. 2019;20:375.

26. Leurs A, Gnemmi V, Lionet A, Renaud L, Gibier JB, Copin MC, et al. Renal pathologic findings in TAFRO syndrome: is there a continuum between thrombotic microangiopathy and membranoproliferative glomerulonephritis? A case report and literature review. Front Immunol. 2019;10:1489.

27. Ozeki T, Tsuji M, Yamamoto J, Shigematsu C, Maruyama S. Thrombotic microangiopathy on kidney biopsy in a patient with TAFRO syndrome. CEN Case Rep. 2018;7:243-7.

28. Mizuno H, Sekine A, Oguro M, Oshima Y, Kawada M, Sumida K, et al. Renal histology in a patient with TAFRO syndrome: a case report. Hum Pathol. 2018;82:258-63.

29. Ganz T. Anemia of inflammation. N Engl J Med. 2019;381:1148-57.

30. Hall P, Cash J. What is the real function of the liver 'function' tests? Ulster Med J. 2012;81:30-6.

31. Masaki Y, Kawabata H, Fujimoto S, Kawano M, Iwaki N, Kotani $\mathrm{T}$, et al. Epidemiological analysis of multicentric and unicentric Castleman disease and TAFRO syndrome in Japan. J Clin Exp Hematop. 2019;59:175-8.

Publisher's Note Springer Nature remains neutral with regard to jurisdictional claims in published maps and institutional affiliations. 


\section{Authors and Affiliations}

Hiroshi Kawabata ${ }^{1}$ (D) Shino Fujimoto ${ }^{1} \cdot$ Tomoyuki Sakai $^{1} \cdot$ Hiroto Yanagisawa $^{1} \cdot$ Toshio Kitawaki $^{2}$. $\mathrm{Kenji} \mathrm{Nara}^{3} \cdot$ Masao Hagihara $^{4} \cdot \mathrm{Hiroshi}$ Yamamoto $^{5}$ - Masakuni Tanimizu ${ }^{6}$. Chikako Kato ${ }^{7}$. Tomoki Origuchi ${ }^{8}$. Kazutaka Sunami ${ }^{9} \cdot$ Yoshitaka Sunami $^{10} \cdot$ Taro Masunari $^{11} \cdot$ Nobuhiko Nakamura $^{12} \cdot$ Masanori Kobayashi $^{13}$. Keiko Yamagami ${ }^{14} \cdot$ Katsuhiro Miura $^{15} \cdot$ Kazue Takai $^{16} \cdot$ Sadao Aoki $^{17} \cdot$ Norifumi Tsukamoto $^{18} \cdot$ Yasufumi Masaki $^{1}$

1 Department of Hematology and Immunology, Kanazawa Medical University, 1-1 Daigaku, Uchinada, Ishikawa-ken 920-0293, Japan

2 Department of Hematology and Oncology, Graduate School of Medicine, Kyoto University, Kyoto, Japan

3 Department of Hematology, Seirei Mikatahara General Hospital, Hamamatsu, Japan

4 Department of Hematology, Eiju General Hospital, Tokyo, Japan

5 First Department of Internal Medicine, Shinshu University School of Medicine, Matsumoto, Japan

6 Department of Internal Medicine, Tottori Municipal Hospital, Tottori, Japan

7 Department of General Internal Medicine, Toyota Kosei Hospital, Toyota, Japan

8 Department of Immunology and Rheumatology, Nagasaki University Graduate School of Biomedical Science, Nagasaki, Japan

9 Department of Hematology, NHO Okayama Medical Center, Okayama, Japan
10 Department of Hematology, Juntendo University Shizuoka Hospital, Izunokuni, Japan

11 Department of Infectious Disease, Chugoku Central Hospital, Fukuyama, Japan

12 Department of Hematology, Gifu University Graduate School of Medicine, Gifu, Japan

13 Kassai Medical Clinic, Osaka, Japan

14 Department of Internal Medicine, Osaka City General Hospital, Osaka, Japan

15 Division of Hematology and Rheumatology, Department of Internal Medicine, Nihon University Itabashi Hospital, Tokyo, Japan

16 Department of Hematology, Niigata City General Hospital, Niigata, Japan

17 Department of Pathophysiology, Faculty of Pharmaceutical Sciences, Niigata University of Pharmacy and Applied Life Sciences, Niigata, Japan

18 Oncology Centre, Gunma University Hospital, Maebashi, Japan 